\title{
Dense subfields of henselian fields, and integer parts*
}

\author{
Franz-Viktor Kuhlmann
}

26. 6.2005

\begin{abstract}
We show that every henselian valued field $L$ of residue characteristic 0 admits a proper subfield $K$ which is dense in $L$. We present conditions under which this can be taken such that $L \mid K$ is transcendental and $K$ is henselian. These results are of interest for the investigation of integer parts of ordered fields. We present examples of real closed fields which are larger than the quotient fields of all their integer parts. Finally, we give rather simple examples of ordered fields that do not admit any integer part and of valued fields that do not admit any subring which is an additive complement of the valuation ring.
\end{abstract}

\section{Introduction}

At the "Logic, Algebra and Arithmetic" Conference, Teheran 2003, Mojtaba Moniri asked the following question: Does every non-archimedean ordered real closed field $L$ admit a proper dense subfield $K$ ? This question is interesting since if such a subfield $K$ admits an integer part $I$ then $I$ is also an integer part for $L$, but the quotient field of $I$ lies in $K$ and is thus smaller than $L$. An integer part of an ordered field $K$ is a discretely ordered subring $I$ with 1 such that for all $a \in K$ there is $r \in I$ such that $r \leq a<r+1$. It follows that the element $r$ is uniquely determined, and in particular that 1 is the least positive element in $I$.

Since the natural valuation of a non-archimedean ordered real closed field $L$ is nontrivial, henselian and has a (real closed) residue field $L v$ of characteristic 0 , the following theorem answers the above question to the affirmative:

*This paper was written while I was a guest of the Equipe Géométrie et Dynamique, Institut Mathématiques de Jussieu, Paris, and of the Equipe Algèbre-Géométrie at the University of Versailles. I gratefully acknowledge their hospitality and support. I was also partially supported by a Canadian NSERC grant and by a sabbatical grant from the University of Saskatchewan. Furthermore I am endebted to the organizers of the conference in Teheran and the members of the IPM and all our friends in Iran for their hospitality and support. I also thank the two referees as well as A. Fornasiero for their careful reading of the paper and their useful suggestions. This paper is dedicated to Salma Kuhlmann who got me interested in the subject and provided the personal contacts that inspired and supported my work. 
Theorem 1 Every henselian non-trivially valued field $(L, v)$ with a residue field of characteristic 0 admits a proper subfield $K$ which is dense in $(L, v)$. This subfield $K$ can be chosen such that $L \mid K$ is algebraic.

Here, density refers to the topology induced by the valuation; that is, $K$ is dense in $(L, v)$ if for every $a \in L$ and all values $\alpha$ in the value group $v L$ of $(L, v)$ there is $b \in K$ such that $v(a-b) \geq \alpha$. In the case of non-archimedean ordered fields with natural (or non-trivial order compatible) valuation, density in this sense is equivalent to density with respect to the ordering.

In the case where the value group $v L$ has a maximal proper convex subgroup, the proof is quite easy, but does in general not render any subfield $K$ such that $L \mid K$ is transcendental. In the case of $v L$ having no maximal proper convex subgroup, the proof is much more involved, but leaves us the choice between $L \mid K$ algebraic or transcendental:

Theorem 2 In addition to the assumptions of Theorem 1, suppose that $v L$ does not have a maximal proper convex subgroup. Then for each integer $n \geq 1$ there is a henselian (as well as a non-henselian) subfield $K$ dense in $L$ such that $\operatorname{trdeg} L \mid K=n$. It can also be chosen such that $\operatorname{trdeg} L \mid K$ is infinite.

To see that such valued fields $(L, v)$ exist, take $x_{i}, i \in \mathbb{N}$, to be a set of algebraically independent elements over an arbitrary field $k$ and define a valuation $v$ on $k\left(x_{i} \mid i \in \mathbb{N}\right)$ by setting $0<v x_{1} \ll v x_{2} \ll \ldots \ll v x_{i} \ll \ldots$; then pass to the henselization of $\left(k\left(x_{i}\right)\right.$ $i \in \mathbb{N}), v$ ). For a more general approach, see Lemma 26 .

Remark 3 A. Fornasiero [F] has shown that every henselian valued field with a residue field of characteristic 0 admits a truncation closed embedding in a power series field with coefficients in the residue field and exponents in the value group (in general, the power series field has to be endowed with a non-trivial factor system). "Truncation closed" means that every truncation of a power series in the image lies again in the image.

It follows that all of the henselian dense subfields admit such truncation closed embeddings. But also the dense non-henselian subfields can be chosen such that they admit truncation closed embeddings. We will sketch the proof in Section 3 (Remarks 25 and 28).

Our construction developed for the proof of Theorem 2 also gives rise to a counterexample to a quite common erroneous application of Hensel's Lemma. A valuation $w$ is called a coarsening of $v$ if its associated valuation ring contains that of $v$. In this case, $v$ induces a valuation $\bar{w}$ on the residue field $K w$ whose valuation ring is simply the image of the valuation ring of $v$ under the residue map associated with $w$. The counterexample proves:

Proposition 4 There are valued fields $(K, v)$ such that $v K$ has no maximal proper convex subgroup, the residue field $(K w, \bar{w})$ is henselian for every non-trivial coarsening $w \neq v$ of $v$, but $(K, v)$ itself is not henselian. 
The proofs of Theorems 1 and 2 and of Proposition 4 are given in Section 3. There, we will also give a more explicit version of Theorem 2 .

In general, the quotient fields of integer parts of an ordered field are smaller than the field. The following theorem will show that there are real closed fields for which the quotient field of every integer part is a proper subfield. If $k$ is any field, then

$$
\operatorname{PSF}(k):=\bigcup_{n \in \mathbb{N}} k\left(\left(t^{\frac{1}{n}}\right)\right)
$$

is called the Puiseux series field over $k$; it is a subfield of the power series field $k\left(\left(t^{\mathbb{Q}}\right)\right)$ with coefficients in $k$ and exponents in $\mathbb{Q}$, which we also simply denote by $k((\mathbb{Q}))$.

Theorem 5 Let $\mathbb{Q}^{\mathrm{rc}}$ denote the field of real algebraic numbers and PSF $\left(\mathbb{Q}^{\mathrm{rc}}\right)$ the Puiseux series field over $\mathbb{Q}^{\mathrm{rc}}$. If $I$ is any integer part of this real closed field, then Quot $I$ is a proper countable subfield of PSF $\left(\mathbb{Q}^{\mathrm{rc}}\right)$ such that the transcendence degree of PSF $\left(\mathbb{Q}^{\mathrm{rc}}\right)$ over Quot $I$ is uncountable. The same holds for the completion of $\operatorname{PSF}\left(\mathbb{Q}^{\mathrm{rc}}\right)$.

This answers a question of M. Moniri. An answer was also given, independently, by L. van den Dries at the conference. A larger variety of such fields is presented in Section 4. On the other hand, there are fields that admit integer parts whose quotient field is the whole field:

Theorem 6 Let $\lambda$ be any cardinal number and $k$ any field of characteristic 0 . Then there exists a henselian valued field $(L, v)$ with residue field $k$ which has the following properties:

a) $L$ contains a $k$-algebra $R$ which is an additive complement of its valuation ring such that Quot $R=L$.

b) At the same time, for each non-zero cardinal number $\kappa \leq \lambda, L$ contains a $k$-algebra $R_{\kappa}$ which is an additive complement of its valuation ring such that $\operatorname{trdeg} L \mid$ Quot $R_{\kappa}=\kappa$.

If in addition $k$ is an archimedean ordered field and $<$ is any ordering on $L$ compatible with $v$ (see Section 2 for this notion), then $(L,<)$ admits an integer part I such that Quot $I=L$. At the same time, for each non-zero cardinal number $\kappa \leq \lambda, L$ admits an integer part $I_{\kappa}$ such that trdeg $L \mid$ Quot $I_{n}=\kappa$.

S. Boughattas $[\mathrm{Bg}]$ has given an example of an ordered (and " $n$-real closed") field which does not admit any integer part. In the last section of our paper, we generalize the approach and consider a notion that comprises integer parts as well as subrings which are additive complements of the valuation ring or of the valuation ideal in a valued field. A subring $R$ of a valued field $(K, v)$ will be called a weak complement (in $K$ ) if it has the following properties:

- $v r \leq 0$ for all $r \in R$,

- for all $a \in K$ there is $r \in R$ such that $v(a-r) \geq 0$. 
Every integer part in a non-archimedean ordered field $K$ is a weak complement with respect to the natural valuation of $K$ (see Lemma 35).

Using a somewhat surprising little observation (Lemma 37) together with a result of [K1] (which is a generalization of a result in [M-S]) we construct examples for valued fields that do not admit any weak complements. From this we obtain ordered fields without integer parts. In particular, we show:

Theorem 7 For every prime field $k$ there are valued rational function fields $k(t, x, y)$ of transcendence degree 3 over the trivially valued subfield $k$ which do not admit any weak complements. There are ordered rational function fields of transcendence degree 3 over $\mathbb{Q}$ which do not admit any integer parts.

There are valued rational function fields of transcendence degree 4 over a trivially valued prime field which do not admit any weak complements, but admit an embedding of their residue field and a cross-section. There are ordered rational function fields of transcendence degree 4 over $\mathbb{Q}$ which do not admit any integer parts, but admit an embedding of their residue field and a cross-section for their natural valuation.

Our example of an $n$-real closed field without integer parts is the $n$-real closure of such an ordered rational function field. It is quite similar to the example given by Boughattas, but in contrast to his example, ours is of finite transcendence degree over $\mathbb{Q}$.

Open Problem: Are there valued fields of transcendence degree $\leq 2$ over a trivially valued ground field that do not admit any weak complements? Are there ordered fields of transcendence degree $\leq 2$ over an archimedean ordered field that do not admit any integer parts? Are there examples of transcendence degree $\leq 3$ with embedding of their residue field and cross-section?

\section{Some preliminaries}

For basic facts from general valuation theory we refer the reader to $[\mathrm{E}],[\mathrm{R}],[\mathrm{W}],[\mathrm{Z}-\mathrm{S}]$, $[\mathrm{K} 2]$. For ramification theory, see $[\mathrm{N}],[\mathrm{E}]$ and $[\mathrm{K} 2]$. In the following, we state some well known facts without proofs.

Take any valued field $(K, v)$. If $v^{\prime}$ is a valuation on the residue field $K v$, then $v \circ v^{\prime}$ will denote the valuation whose valuation ring is the subring of the valuation ring of $v$ consisting of all elements whose $v$-residue lies in the valuation ring of $v^{\prime}$. (Note that we identify equivalent valuations.) While $v \circ v^{\prime}$ does actually not mean the composition of $v$ and $v^{\prime}$ as mappings, this notation is used because in fact, up to equivalence the place associated with $v \circ v^{\prime}$ is indeed the composition of the places associated with $v$ and $v^{\prime}$.

Every convex subgroup $\Gamma$ of $v K$ gives rise to a coarsening $v_{\Gamma}$ of $v$ such that $v_{\Gamma} K$ is isomorphic to $v K / \Gamma$. As mentioned in the introduction, $v$ induces a valuation $\bar{v}_{\Gamma}$ on the residue field $K v_{\Gamma}$. We then have that $v=v_{\Gamma} \circ \bar{v}_{\Gamma}$. The value group $\bar{v}_{\Gamma}\left(K v_{\Gamma}\right)$ of $\bar{v}_{\Gamma}$ is 
isomorphic to $\Gamma$, and its residue field $\left(K v_{\Gamma}\right) \bar{v}_{\Gamma}$ is isomorphic to $K v$. Every coarsening $w$ of $v$ is of the form $v_{\Gamma}$ for some convex subgroup $\Gamma$ of $v K$.

If $a$ is an element of the valuation $\operatorname{ring} \mathcal{O}_{v}$ of $v$ on $K$, then $a v$ will denote the image of $a$ under the residue map associated with the valuation $v$. This map is a ring homomorphism from $\mathcal{O}_{v}$ onto the residue field $K v$. It is only unique up to equivalence, i.e., up to composition with an isomorphism from $K v$ to another field (and so the residue field $K v$ is only unique up to isomorphism). If $w$ is a coarsening of $v$, that is, $\mathcal{O}_{v}$ contains the valuation ring $\mathcal{O}_{w}$ of $v$ on $L$, then the residue map $\mathcal{O}_{w} \ni a \mapsto a w \in K w$ can be chosen such that it extends the residue map $\mathcal{O}_{v} \ni a \mapsto a v \in K v$.

An ordering $<$ on a valued field $(K, v)$ is said to be compatible with the valuation $v$ (and $v$ is compatible with $<$ ) if

$$
\forall x, y \in K: \quad 0<x \leq y \Rightarrow v x \geq v y .
$$

This holds if and only if the valuation ring of $v$ is a convex subset of $(K,<)$. This in turn holds if and only if $<$ induces an ordering on the residue field $K v$. We will need the following well-known facts (cf. [P]):

Lemma 8 Take any valued field $(K, v)$. Every ordering $<_{r}$ on $K v$ can be lifted to an ordering $<$ on $K$ which is compatible with $v$ and induces $<_{r}$ on $K v$ (that is, if $a, b$ are elements of the valuation ring of $v$ such that $a<b$, then $a v=b v$ or av $\left.<_{r} b v\right)$.

Lemma 9 If an ordering of a field $K$ is compatible with the valuation $v$ of $K$, then $v$ extends to a valuation of the real closure $K^{\mathrm{rc}}$ of $(K,<)$, which is still compatible with the ordering on $K^{\mathrm{rc}}$. This extension is henselian, its value group $v K^{\mathrm{rc}}$ is the divisible hull of $v K$, and its residue field $K^{\mathrm{rc}} v$ is the real closure of $K v$ (with respect to the induced ordering on $K v$ ).

A compatible valuation of an ordered field $(K,<)$ is called the natural valuation of $(K,<)$ if its residue field is archimedean ordered. The natural valuation is uniquely determined, and every compatible valuation is a coarsening of the natural valuation.

Take any valued field $(K, v)$ and a finite extension $L \mid K$. Then the following fundamental inequality holds:

$$
n \geq \sum_{i=1}^{\mathrm{g}} \mathrm{e}_{i} \mathrm{f}_{i},
$$

where $n=[L: K]$ is the degree of the extension, $v_{1}, \ldots, v_{\mathrm{g}}$ are the distinct extensions of $v$ from $K$ to $L, \mathrm{e}_{i}=\left(v_{i} L: v K\right)$ are the respective ramification indices, and $\mathrm{f}_{i}=\left[L v_{i}: K v\right]$ are the respective inertia degrees. Note that the extension of $v$ from $K$ to $L$ is unique (i.e., $\mathrm{g}=1)$ if and only if $(K, v)$ is henselian (which by definition means that $(K, v)$ satisfies Hensel's Lemma). The following are easy consequences: 
Lemma 10 If $L \mid K$ is a finite extension and $v$ is a valuation on $L$, then $[L: K] \geq(v L$ : $v K)$ and $[L: K] \geq[L v: K v]$.

Corollary 11 Let $L \mid K$ be an algebraic extension and $v$ a valuation on $L$. Then $v L / v K$ is a torsion group and the extension $L v \mid K v$ of residue fields is algebraic. If $v$ is trivial on $K$ (i.e., $v K=\{0\}$ ), then $v$ is trivial on $L$.

An extension $(K, v) \subseteq(L, v)$ of valued fields is called immediate if the canonical embeddings of $v K$ in $v L$ and of $K v$ in $L v$ are onto. We have:

Lemma 12 If $K$ is dense in $(L, v)$, then $(K, v) \subseteq(L, v)$ is an immediate extension.

Proof: If $a \in L$ and $b \in K$ such that $v(a-b)>v a$, then $v a=v b \in v K$. If $a \in L$ such that $v a=0$ and $b \in K$ such that $v(a-b)>0$, then $a v=b v \in K v$.

The following is a well known consequence of the so-called "Lemma of Ostrowski":

Lemma 13 If a valued field $(L, v)$ is an immediate algebraic extension of a henselian field $(K, v)$ of residue characteristic 0 , then $L=K$.

Lemma 14 The henselization $K^{h}$ of a valued field $(K, v)$ (which is unique up to valuation preserving isomorphism over $K$ ) is an immediate extension and can be chosen in every henselian valued extension field of $(K, v)$.

Lemma 15 An algebraic extension of a henselian valued field, equipped with the unique extension of the valuation, is again henselian.

Lemma 16 Let $(L, v)$ be any field and $v=w \circ \bar{w}$ where $w$ is non-trivial. Take any subfield $L_{0}$ of $L$. Then $L_{0}$ is dense in $(L, v)$ if and only if $L_{0}$ is dense in $(L, w)$.

Lemma 17 Let $(K, v)$ be any field and $v=w \circ \bar{w}$. Then $(K, v)$ is henselian if and only if $(K, w)$ and $(K w, \bar{w})$ are.

Corollary 18 Let $(K, v)$ be any field and $v=w \circ \bar{w}$. If $(K w, \bar{w})$ is henselian, then the henselization of $(K, v)$ is equal to the henselization of $(K, w)$ (as fields).

The value group $v K$ of a valued field $(K, v)$ is archimedean if it is embeddable in the ordered additive group of the reals. This holds if and only if every convex subgroup of $v K$ is equal to $\{0\}$ or to $v K$.

Lemma 19 If $(K, v)$ is a valued field such that $v K$ is archimedean, then $K$ is dense in its henselization. In particular, the completion of $(K, v)$ is henselian. 
The following result is an easy application of Hensel's Lemma:

Lemma 20 Take $(K, v)$ to be a henselian valued field of residue characteristic char $K v=$ 0 . Take any subfield $K_{0}$ of $K$ on which $v$ is trivial. Then there is a subfield $K^{\prime}$ of $K$ containing $K_{0}$ and such that $v$ is trivial on $K^{\prime}$ and the residue map associated with $v$ induces an isomorphism from $K^{\prime}$ onto $K v$. If $K v \mid K_{0} v$ is algebraic, then so is $K^{\prime} \mid K_{0}$.

A field $K^{\prime}$ as in this lemma is called a field of representatives for the residue field $K v$.

Proposition 21 a) Take a non-empty set $T$ of elements algebraically independent over $K$ and a finite extension $F$ of $K(T)$. Then no non-trivial valuation on $F$ is henselian. In particular, no non-trivial valuation on an algebraic function field (of transcendence degree at least one) is henselian.

b) Fix $n \in \mathbb{N}$, take $K(T)$ as in a) and take $F$ to be the closure of $K(T)$ under successive adjunction of roots of polynomials of degree $\leq n$. Then no non-trivial valuation on $F$ is henselian.

Proof: Choosing any $t \in T$ and replacing $K$ by $K(T \backslash\{t\})$, we may assume in parts a) and b) that $T$ consists of a single element, i.e., $\operatorname{trdeg} F \mid K=1$.

Take any non-trivial valuation on $F$. We show that there is some $x \in K(T)$ such that $v x>0$ and $x$ is transcendental over $K$. Assume first that $v$ is trivial on $K$. Since $v$ is non-trivial on $F$ and $F \mid K(T)$ is algebraic, Corollary 11 shows that $v$ is non-trivial on $K(T)$. Hence there must be some $x \in K(T)$ such that $v x \neq 0$. Replacing $x$ by $x^{-1}$ if necessary, we may assume that $v x>0$. It follows that $x \notin K$, so $x$ is transcendental over $K$.

Now assume that $v$ is not trivial on $K$, and take an arbitrary $x \in K(T)$ transcendental over $K$. If $v x>0$, we are done. If $v x<0$, we replace it by $x^{-1}$ and we are done again. If $v x=0$, we pick some $c \in K$ such that $v c>0$. Then $v c x>0$ and $c x$ is transcendental over $K$, hence replacing $x$ by $c x$ finishes the proof of our claim.

Pick any positive integer $q$ such that $q$ is not divisible by the characteristic $p:=$ char $K v$ of the residue field $K v$. By Hensel's Lemma, any henselian extension of $K(x)$ will contain a $q$-th root of the 1-unit $y:=1+x$. We wish to show that any algebraic extension of $K(x)$ containing such a $q$-th root must be of degree at least $q$ over $K(x)$. A valuation theoretical proof for this fact reads as follows. Take the $y$-adic valuation $v_{y}$ on $K(x)=K(y)$. Then $v_{y} y$ is the least positive element in the value group $v_{y} K(x) \simeq \mathbb{Z}$, and any $q$-th root $b$ of $y$ will have $v_{y}$-value $\frac{1}{q} v_{y} y$. This shows that $\left(v_{y} K(x)(b): v_{y} K(x)\right) \geq q$. By the fundamental inequality, it follows that $[K(x, b): K(x)] \geq\left(v_{y} K(x, b): v_{y} K(x)\right) \geq q$.

Proof of part a): Since $\operatorname{trdeg} F \mid K=1, x \in K(T)$ is transcendental over $K$ and $F \mid K$ is finite, also $F \mid K(x)$ is finite. Pick $q>[F: K(x)]$ not divisible by $p$. Then it follows that $F$ does not contain a $q$-th root of $y$, and so $(F, v)$ cannot be henselian. 
Proof of part b): This time, we still have that $K(T) \mid K(x)$ is finite. Pick a prime $q>\max \{n,[K(T): K(x)]\}, q \neq p$. For every element $\alpha$ in the value group $v_{y} F$ there is an integer $e$ which is a product of positive integers $\leq n$ such that $e \alpha \in v_{y} K(T)$. Further, there is a positive integer $e^{\prime}$ such that $e^{\prime} e \alpha \in v_{y} K(x)$. On the other hand, by our choice of $q$, it does not divide $e^{\prime} e$. Since the order of the value $\frac{1}{q} v_{y} y$ modulo $v_{y} K(x)$ is $q$, it follows that this value does not lie in $v_{y} F$. Hence again, $(F, v)$ cannot be henselian.

Proposition 22 Take $(L, v)$ to be a henselian field of residue characteristic char $L v=0$, and $K$ a subfield of $L$ such that $L \mid K$ is algebraic. Then $K$ admits an algebraic extension $L_{0}$ inside of $L$ such that the extension of $v$ from $K$ to $L_{0}$ is unique, $L_{0}$ is linearly disjoint over $K$ from the henselization $K^{h}$ of $K$ in $L$, and $L=L_{0} \cdot K^{h}=L_{0}^{h}$.

Proof: $\quad$ Take any subextension $L_{0} \mid K$ of $L \mid K$ maximal with the property that the extension of $v$ from $K$ to $L_{0}$ is unique. By general ramification theory it follows that $L_{0} \mid K$ is linearly disjoint from $K^{h} \mid K$ and that $L_{0}^{h}=L_{0} \cdot K^{h}$. We only have to show that $L_{0}^{h}=L$. Note that $L \mid L_{0}^{h}$ is algebraic since already $L \mid K$ is algebraic,

Let us show that $L_{0} v=L v$. If this is not the case, then there is be some element $\zeta \in L v \backslash L_{0} v$. By Corollary $11, L v \mid L_{0} v$ is algebraic. Let $g \in L_{0} v[X]$ be the minimal polynomial of $\zeta$ over $L_{0} v$. Since char $K v=0, g$ is separable. We choose some monic polynomial $f$ with integral coefficients in $L_{0}$ whose reduction modulo $v$ is $g$; it follows that $\operatorname{deg} f=\operatorname{deg} g$. Since $\zeta$ is a simple root of $g$, it follows from Hensel's Lemma that the henselian field $(L, v)$ contains a root $z$ of $f$ whose residue is $\zeta$. We have

$$
\left[L_{0}(z): L_{0}\right] \leq \operatorname{deg} f=\operatorname{deg} g=\left[L_{0} v(\zeta): L_{0} v\right] \leq\left[L_{0}(z) v: L_{0} v\right] \leq\left[L_{0}(z): L_{0}\right],
$$

where the last inequality follows from Lemma 10. We conclude that $\left[L_{0}(z): L_{0}\right]=$ $\left[L_{0}(z) v: L_{0} v\right]$. From the fundamental inequality it follows that the extension of $v$ from $L_{0}$ (and hence also from $K$ ) to $L_{0}(z)$ is unique. But this contradicts the maximality of $L_{0}$. Hence, $L_{0} v=L v$.

Next, let us show that $v L_{0}=v L$. If this is not the case, then there is some $\alpha \in v L \backslash v L_{0}$. By Corollary 11, $v L / v L_{0}$ is a torsion group and hence there is some $n>1$ such that $n \alpha \in v L_{0}$. We choose $n$ minimal with this property, so that $\left(v L_{0}+\alpha \mathbb{Z}: v L_{0}\right)=n$. Further, we pick some $a \in L$ such that $v a=\alpha$. Since $n \alpha \in v L_{0}$, there is some $d \in L_{0}$ such that $v d=n \alpha=v a^{n}$. It follows that $v a^{n} / d=0$, and since we have already shown that $L v=L_{0} v$, we can choose some $c \in L_{0}$ such that $\left(a^{n} / c d\right) v=1$. Consequently, the reduction of $X^{n}-a^{n} / c d$ modulo $v$ is the polynomial $X^{n}-1$, which admits 1 as a simple root since char $K v=0$. Hence by Hensel's Lemma, $X^{n}-a^{n} / c d$ admits a root $b$ in the henselian field $(L, v)$. For $z:=\frac{a}{b}$ it follows that

$$
n v z=v \frac{a^{n}}{b^{n}}=v c d=v d=n \alpha,
$$


which shows that $\alpha=v z \in v L_{0}(z)$. We have

$$
\left[L_{0}(z): L_{0}\right] \leq n=\left(v L_{0}+\alpha \mathbb{Z}: v L_{0}\right) \leq\left(v L_{0}(z): v L_{0}\right) \leq\left[L_{0}(z): L_{0}\right]
$$

where again the last inequality follows from Lemma 10. We conclude that $\left[L_{0}(z): L_{0}\right]=$ $\left(v L_{0}(z): v L_{0}\right)$. From the fundamental inequality it follows that the extension of $v$ from $L_{0}$ (and hence also from $K$ ) to $L_{0}(z)$ is unique. But this again contradicts the maximality of $L_{0}$. Hence, $v L_{0}=v L$.

We have shown that $v L=v L_{0}$ and $L v=L_{0} v$. Hence, $v L=v L_{0}^{h}$ and $L v=L_{0}^{h} v$. As $L \mid L_{0}$ is algebraic, the same is true for $L \mid L_{0}^{h}$. Since the residue field characteristic of $(L, v)$ is zero, Lemma 13 shows that $L=L_{0}^{h}$. This concludes our proof.

\section{Dense subfields}

In this section we prove the existence of proper dense subfields of henselian fields with residue characteristic 0 .

Proposition 23 Take a henselian valued field $(L, v)$ such that $v L$ admits a maximal proper convex subgroup $\Gamma$. Assume that char $L v_{\Gamma}=0$. Then $L$ admits a proper dense subfield $L_{0}$ such that $L \mid L_{0}$ is algebraic.

Proof: By Lemma 16 it suffices to find a subfield $L_{0}$ which is dense in $L$ with respect to $v_{\Gamma}$, and such that $L \mid L_{0}$ is algebraic. By Lemma $17,\left(L, v_{\Gamma}\right)$ is henselian. Since char $L v_{\Gamma}=0$ and hence char $L=0, L$ contains $\mathbb{Q}$ and $v_{\Gamma}$ is trivial on $\mathbb{Q}$. Pick a transcendence basis $T$ of $L \mid \mathbb{Q}$. Since $v_{\Gamma}$ is non-trivial on $L, T$ is non-empty. We infer from Lemma 21 that $\left(\mathbb{Q}(T), v_{\Gamma}\right)$ is not henselian. By Proposition 22 , there is an algebraic extension $L_{0}$ of $\mathbb{Q}(T)$ within $L$ such that $L_{0}$ is linearly disjoint over $\mathbb{Q}(T)$ from the $v_{\Gamma^{-h}}$-nselization $\mathbb{Q}(T)^{h}$ of $\mathbb{Q}(T)$ in $L$, and $L=L_{0} \cdot \mathbb{Q}(T)^{h}=L_{0}^{h}$. Since $\left(\mathbb{Q}(T), v_{\Gamma}\right)$ is not henselian, $\mathbb{Q}(T)^{h} \mid \mathbb{Q}(T)$ is a proper extension. By the linear disjointness, the same holds for $L \mid L_{0}$. As $\Gamma$ is the maximal proper convex subgroup of $v L, v_{\Gamma} L \simeq v L / \Gamma$ must be archimedean. Thus by Lemma $19,\left(L_{0}, v_{\Gamma}\right)$ lies dense in its henselization $\left(L, v_{\Gamma}\right)$. Hence by Lemma $16,\left(L_{0}, v\right)$ lies dense in its henselization $(L, v)$. Since $L \mid \mathbb{Q}(T)$ is algebraic, so is $L \mid L_{0}$.

In certain cases, even if $v$ has a coarsest non-trivial coarsening, there will also be dense subfields $K$ such that $L \mid K$ is transcendental. For instance, this is the case for $L=k((t))$ equipped with the $t$-adic valuation $v_{t}$, where a subfield is dense in $L$ as soon as it contains $k(t)$. On the other hand, the henselization $k(t)^{h}$ of $k(t)$ w.r.t. $v_{t}$ admits $k(t)$ as a proper dense subfield, and the extension $k(t)^{h} \mid K$ is algebraic for every subfield $K$ which is dense in $k(t)^{h}$. More generally, the following holds: 
Proposition 24 Suppose that $(L, v)$ is a valued field and that $v$ is trivial on the prime field $k$ of $L$. If

$$
\operatorname{trdeg} L\left|k=\operatorname{dim}_{\mathbb{Q}}(\mathbb{Q} \otimes v L)+\operatorname{trdeg} L v\right| k<\infty,
$$

then $L \mid K$ is algebraic for every dense subfield $K$.

Proof: If $K$ is a dense subfield, then by Lemma $12,(L \mid K, v)$ is an immediate extension. Hence,

$\operatorname{trdeg} K\left|k \geq \operatorname{dim}_{\mathbb{Q}}(\mathbb{Q} \otimes v K)+\operatorname{trdeg} K v\right| k=\operatorname{dim}_{\mathbb{Q}}(\mathbb{Q} \otimes v L)+\operatorname{trdeg} L v|k=\operatorname{trdeg} L| k$,

whence $\operatorname{trdeg} K|k=\operatorname{trdeg} L| k$, showing that $L \mid K$ is algebraic.

Note that if $(L, v)$ is a valued field with a subfield $L_{0}$ on which $v$ is trivial, and if $\operatorname{trdeg} L \mid L_{0}<\infty$, then in general,

$$
\operatorname{trdeg} L\left|L_{0} \geq \operatorname{dim}_{\mathbb{Q}}(\mathbb{Q} \otimes v L)+\operatorname{trdeg} L v\right| L_{0} .
$$

This is a special case of the so-called "Abhyankar inequality". For a proof, see $[\mathrm{Br}]$, Chapter VI, $\S 10.3$, Theorem 1 . Note that $\mathbb{Q} \otimes v L$ is the divisible hull of $v L$, and $\operatorname{dim}_{\mathbb{Q}}(\mathbb{Q} \otimes$ $v L$ ) is the maximal number of rationally independent elements in $v L$.

Remark 25 It can be shown that if char $L v=0$, then the dense subfield $L_{0}$ in Proposition 23 can always be constructed in such a way that it admits a truncation closed embedding into a power series field. The idea is as follows. Since $(L, v)$ is henselian, we can use Lemma 20 to find a field $k$ of representatives in $L$ for the residue field $L v$. Then we can choose a twisted cross-section as in [F]. The field $L_{1}$ generated over $k$ by the image of the cross-section admits a truncation closed embedding in $k((v L))$ with a suitable factor system, and this embedding $\iota$ can be extended to a truncation closed embedding of $(L, v)$ in $k((v L))$ (cf. [F]). It is easy to show that $L_{\Gamma}:=\iota^{-1}(\iota L \cap k((\Gamma)))$ is a field of representatives for the residue field $L v_{\Gamma}$ in $\left(L, v_{\Gamma}\right)$, and that $\iota$ induces a truncation closed embedding of $L_{\Gamma}$ in $k((\Gamma)) \subset k((v L))$. This can be extended to a truncation closed embedding of $L_{2}:=L_{1} . L_{\Gamma}$ which is obtained from $L_{\Gamma}$ by adjoining the image of the cross-section. We note that $\left(L, v_{\Gamma}\right)$ is an immediate extension of $\left(L_{2}, v_{\Gamma}\right)$. If this extension is algebraic, then $L$ is also algebraic over the henselization of $L_{2}$ (with respect to $v_{\Gamma}$ ), and by Lemma 13 , the two fields must be equal. That shows that $L_{2}$ is dense in $\left(L, v_{\Gamma}\right)$ and hence in $(L, v)$, and we can take $L_{0}=L_{2}$.

If $L \mid L_{2}$ is transcendental, we take a transcendence basis $S$ of $L \mid L_{2}$ and pick $s \in S$. Then one shows as before that $L$ is the henselization of $L_{2}(S)$, and also of the larger field $L_{0}:=L_{2}(S \backslash\{s\})^{h}(s)$. Again, $L_{0}$ is dense in $(L, v)$. Following [F], $L_{2}(S \backslash\{s\})^{h}$ admits a truncation closed embedding in $k((v L))$. As $(L, v)$ is immediate over $\left(L_{2}, v\right)$, it is also immediate over $L_{2}(S \backslash\{s\})^{h}$. Therefore, $s$ is the limit of a pseudo Cauchy sequence in $L_{2}(S \backslash\{s\})^{h}$ without a limit in this field. As the field is henselian of residue characteristic 0 , this pseudo Cauchy sequence is of transcendental type. Now $[\mathrm{F}]$ shows that the truncation closed embedding can be extended to $L_{0}$. 
Now we turn to the case where $v L$ admits no maximal proper convex subgroup, i.e., $v$ admits no coarsest non-trivial coarsening. Such valued fields exist:

Lemma 26 Take any regular cardinal number $\lambda$ and any field $k$. Then there is a valued field $(L, v)$ with residue field $k$ and such that $\lambda$ is the cofinality of the set of all proper convex subgroups of $v L$, ordered by inclusion.

Proof: $\quad$ Take $J$ to be the set of all ordinal numbers $<\lambda$, endowed with the reverse of the usual ordering. Choose any archimedean ordered abelian group $\Gamma$. Then take $G$ to be the ordered Hahn product $\mathbf{H}_{J} \Gamma$ with index set $J$ and components $\Gamma$ (see $[\mathrm{Fu}]$ or $[\mathrm{KS}]$ for details on Hahn products). Then the set of all proper convex subgroups of $G$, ordered by inclusion, has order type $\lambda$ and hence has cofinality $\lambda$. Now take $(L, v)$ to be the power series field $k((G))$ with its canonical valuation.

Note that if $v L$ admits no maximal proper convex subgroup, then $v L$ is the union of its proper convex subgroups. Indeed, if $\alpha \in v L$, then the smallest convex subgroup $C$ of $v L$ that contains $\alpha$ (= the intersection of all convex subgroups containing $\alpha$ ) admits a largest convex subgroup, namely the largest convex subgroup of $v L$ that does not contain $\alpha$ (= the union of all convex subgroups not containing $\alpha$ ). Therefore $C \neq v L$, showing that $C$ is a proper convex subgroup containing $\alpha$.

Proposition 27 Take a henselian valued field $(L, v)$ such that $v L$ admits no maximal proper convex subgroup. Assume that char $L=0$. Then $L$ admits a proper dense subfield $K$ such that $L \mid K$ is algebraic. If $\kappa>0$ is any cardinal number smaller than or equal to the cofinality of the set of convex subgroups of $v L$ ordered by inclusion, then there is also a henselian (as well as a non-henselian) subfield $K$ dense in $L$ such that $\operatorname{trdeg} L \mid K=\kappa$.

Proof: It suffices to prove that there is a subfield $K$ dense in $L$ such that the transcendence degree of $L \mid K$ is equal to the cofinality $\lambda$ of the set of convex subgroups of $v L$. This is seen as follows. Take a transcendence basis $T$ of $L \mid K$. If $\kappa$ is a cardinal number $\leq \lambda$, then take a subset $T_{\kappa}$ of $T$ of cardinality $\kappa$. Then $K_{\kappa}:=K\left(T \backslash T_{\kappa}\right)$ is dense in $L$ because it contains $K$; furthermore, $\operatorname{trdeg} L \mid K_{\kappa}=\kappa$. We may always, even in the case of $\kappa=\lambda$, choose $T_{\kappa} \neq T$. Then by part a) of Proposition $21,\left(K_{\kappa}, v\right)$ is not henselian. In particular, $(K(T), v)$ is not henselian and thus, $K(T)$ is a proper subfield of $L$ such that $L \mid K(T)$ is algebraic. If $\kappa \neq 0$, then $L \mid K_{\kappa}$ will be transcendental. By Lemma 14, the henselian field $L$ contains the henselization $K_{\kappa}^{h}$ of $K_{\kappa}$. Since it is an algebraic extension of $K_{\kappa}$, we have trdeg $L\left|K_{\kappa}^{h}=\operatorname{trdeg} L\right| K_{\kappa}=\kappa$, and it is dense in $L$, too.

To illustrate the idea of our proof, we first show that there is a dense subfield $K$ such that $\operatorname{trdeg} L \mid K>0$. We choose a convex subgroup $C_{0}$ of $v L$ as follows. If char $L v=0$, then we set $C_{0}=\{0\}$. If char $L v=p>0$, then we observe that $0 \neq p \in L$ since char $L=0$, so we may take $C_{0}$ to be the smallest proper convex subgroup that contains 
$v p$. We let $w_{0}=v_{C_{0}}$ be the coarsening of $v$ associated with $C_{0}$. We have $w_{0}=v$ if char $L v=0$. Since $C_{0}$ is a proper convex subgroup, $w_{0}$ is a non-trivial valuation.

Let $\lambda$ be the cofinality of the set of all proper convex subgroups of $v L$, ordered by inclusion. Starting from $C_{0}$, we pick a strictly ascending cofinal sequence of convex subgroups $C_{\nu}, \nu<\lambda$, in this set. We denote by $w_{\nu}$ the coarsening of $v$ which corresponds to $C_{\nu}$.

By Lemma 20 there is a field $K_{0}^{\prime}$ of representatives for $L w_{0}$ in $L$. We pick a transcendence basis $T_{0}=\left\{t_{0, \mu} \mid \mu<\kappa_{0}\right\}$ of $K_{0}^{\prime} \mid \mathbb{Q}$, where $\kappa_{0}$ is the transcendence degree of $L w_{0} \mid \mathbb{Q}$. Then we proceed by induction on $\nu<\lambda$. Suppose we have already constructed a field $K_{\nu}^{\prime}$ of representatives of $L w_{\nu}$ and a transcendence basis $\bigcup_{\nu^{\prime} \leq \nu} T_{\nu^{\prime}}$ for it. By Lemma 20, $K_{\nu}^{\prime}$ can be extended to a field $K_{\nu+1}^{\prime}$ of representatives of $L w_{\nu+1}$, and we choose a transcendence basis $T_{\nu+1}=\left\{t_{\nu+1, \mu} \mid \mu<\kappa_{\nu+1}\right\}$ of $K_{\nu+1}^{\prime} \mid K_{\nu}^{\prime}$. Having constructed $K_{\nu}^{\prime}, \nu<\lambda^{\prime}$ for some limit ordinal $\lambda^{\prime} \leq \lambda$, we set $K_{\lambda^{\prime}}^{*}=\bigcup_{\nu<\lambda^{\prime}} K_{\nu}^{\prime}$. Again by Lemma 20, $K_{\lambda^{\prime}}^{*}$ can be extended to a field of representatives $K_{\lambda^{\prime}}^{\prime}$ of $L w_{\lambda^{\prime}}$, and we choose a transcendence basis $T_{\lambda^{\prime}}=\left\{t_{\lambda^{\prime}, \mu} \mid \mu<\kappa_{\lambda^{\prime}}\right\}$ of $K_{\lambda^{\prime}}^{\prime} \mid K_{\lambda^{\prime}}^{*}$. Note that $T_{\lambda^{\prime}}$ may be empty.

We set $K^{\prime}=\bigcup_{\nu<\lambda} K_{\nu}^{\prime}$ and show that $K^{\prime}$ is dense in $L$. Take any $a \in L$ and $\alpha \in v L$. Then there is some $\nu<\lambda$ such that $\alpha \in C_{\nu}$. By construction, $K^{\prime}$ contains a field of representatives for $L w_{\nu}$. Hence there is some $b \in K^{\prime}$ such that $a w_{\nu}=b w_{\nu}$, meaning that $w_{\nu}(a-b)>0$ and thus, $v(a-b)>\alpha$. This proves our claim. Hence if $\operatorname{trdeg} L \mid K^{\prime}>0$, we set $K=K^{\prime}$ and we are done showing the existence of a subfield $K$ with $\operatorname{trdeg} L \mid K>0$. But it may well happen that $L \mid K^{\prime}$ is algebraic, or even that $L=K^{\prime}$. In this case, we construct a subfield $K$ of $K^{\prime}$ as follows.

Note that for all $\nu<\lambda,\left(K_{\nu}^{\prime}, v\right)$ is henselian. Indeed, it is isomorphic (by the place associated with $\left.w_{\nu}\right)$ to $\left(L w_{\nu}, \bar{w}_{\nu}\right)$, where $\bar{w}_{\nu}$ is the valuation induced by $v$ on $L w_{\nu}$; since $(L, v)$ is henselian, Lemma 17 shows that the same is true for $\left(L w_{\nu}, \bar{w}_{\nu}\right)$ and hence for $\left(K_{\nu}^{\prime}, v\right)$. Again from Lemma 17 it follows that $\left(K_{\nu}^{\prime}, w_{\mu}\right)$ is henselian for all $\mu<\lambda$. Note that $w_{\mu}$ is non-trivial on $K_{\nu}^{\prime}$ only for $\mu<\nu$, and in this case, $K_{\nu}^{\prime} w_{\mu}=L w_{\mu}$ since $K_{\nu}^{\prime}$ contains the field $K_{\mu}^{\prime}$ of representatives for $L w_{\mu}$.

Note further that for all $\nu<\lambda$ and all $\mu<\kappa_{\nu}, w_{\nu} t_{\nu, \mu}=0$. On the other hand, after multiplication with suitable elements in $K_{\nu+1}$ we may assume that $w_{\nu} t_{\nu+1, \mu}>0$ for all $\mu<\kappa_{\nu+1}$.

We will now construct inside of $K^{\prime}$ a chain (ordered by inclusion) of subfields $K_{\nu} \subset K_{\nu}^{\prime}$ $(\nu<\lambda)$ such that each $K_{\nu}$ is a field of representatives for $L w_{\nu}$ and contains the element $t_{0,0}-t_{\nu+1,0}$, but not the element $t_{0,0}$.

Since $T_{0}=\left\{t_{0, \mu} \mid \mu<\kappa_{0}\right\}$ is a transcendence basis of $K_{0}^{\prime} \mid \mathbb{Q}$, Lemma 11 shows that the residue field $K_{1}^{\prime} w_{0}=K_{0}^{\prime} w_{0}$ is algebraic over $\mathbb{Q}\left(t_{0, \mu} \mid \mu<\kappa_{0}\right) w_{0}$. Because $\left(t_{0,0}-t_{1,0}\right) w_{0}=$ $t_{0,0} w_{0}$ by construction, the latter field is equal to $\mathbb{Q}\left(t_{0,0}-t_{1,0}, t_{0, \mu} \mid 1 \leq \mu<\kappa_{0}\right) w_{0}$. Since char $K_{1}^{\prime} w_{0}=0$, we can use Lemma 20 to find inside of the henselian field $\left(K_{1}^{\prime}, w_{0}\right)$ an algebraic extension $K_{0}$ of $\mathbb{Q}\left(t_{0,0}-t_{1,0}, t_{0, \mu} \mid 1 \leq \mu<\kappa_{0}\right)$ which is a field of representatives for $K_{1}^{\prime} w_{0}=L w_{0}$. Note that $t_{0,0}$ is transcendental over $\mathbb{Q}\left(t_{0,0}-t_{1,0}, t_{0, \mu} \mid 1 \leq \mu<\kappa_{0}\right)$ and therefore, $t_{0,0} \notin K_{0}$, but $t_{0,0}-t_{1,0} \in K_{0}$. 
Suppose we have already constructed all fields $K_{\mu}$ for $\mu \leq \nu$, where $\nu$ is some ordinal $<$ $\lambda$. Since $T_{\nu+1}=\left\{t_{\nu+1, \mu} \mid \mu<\kappa_{\nu+1}\right\}$ is a transcendence basis of $K_{\nu+1}^{\prime} \mid K_{\nu}^{\prime}$, Lemma 11 shows that the residue field $K_{\nu+2}^{\prime} w_{\nu+1}=K_{\nu+1}^{\prime} w_{\nu+1}$ is algebraic over $K_{\nu}\left(t_{\nu+1, \mu} \mid \mu<\kappa_{\nu+1}\right) w_{\nu+1}$. Because $\left(t_{\nu+1,0}-t_{\nu+2,0}\right) w_{\nu+1}=t_{\nu+1,0} w_{\nu+1}$ by construction, the latter field is equal to $K_{\nu}\left(t_{\nu+1,0}-t_{\nu+2,0}, t_{\nu+1, \mu} \mid 1 \leq \mu<\kappa_{\nu+1}\right) w_{\nu+1}$. Since char $K_{\nu+2}^{\prime} w_{\nu+1}=0$, we can use Lemma 20 to find inside of the henselian field $\left(K_{\nu+2}^{\prime}, w_{\nu+1}\right)$ an algebraic extension $K_{\nu+1}$ of $K_{\nu}\left(t_{\nu+1,0}-t_{\nu+2,0}, t_{\nu+1, \mu} \mid 1 \leq \mu<\kappa_{\nu+1}\right)$ which is a field of representatives for $K_{\nu+2}^{\prime} w_{\nu+1}=$ $L w_{\nu+1}$. Since $t_{0,0}-t_{\nu+1,0}, t_{\nu+1,0}-t_{\nu+2,0} \in K_{\nu+1}$ we have that $t_{0,0}-t_{\nu+2,0} \in K_{\nu+1}$. Again, $t_{0,0} \notin K_{\nu+1}$ as $t_{0,0}$ is transcendental over $K_{\nu}\left(t_{\nu+1,0}-t_{\nu+2,0}, t_{\nu+1, \mu} \mid 1 \leq \mu<\kappa_{\nu+1}\right)$.

Suppose we have already constructed all fields $K_{\nu}$ for $\nu<\lambda^{\prime}$, where $\lambda^{\prime}$ is some limit ordinal $\leq \lambda$. We note that $t_{0,0} \notin \bigcup_{\nu<\lambda^{\prime}} K_{\nu}=: K_{\lambda^{\prime}}^{* *}$. But $K_{\lambda^{\prime}}^{* *}\left(t_{0,0}\right)$ contains the entire transcendence basis of $K_{\lambda^{\prime}}^{*} \mid \mathbb{Q}$ because $t_{0,0}-t_{\nu+1,0} \in K_{\nu}$ for every $\nu<\lambda^{\prime}$ (recall that $K_{\lambda^{\prime}}^{*}$ is the field we constructed above before constructing $K_{\lambda^{\prime}}^{\prime}$ ). It follows that $T_{\lambda^{\prime}} \cup\left\{t_{0,0}\right\}$ is a transcendence basis of $K_{\lambda^{\prime}}^{\prime} \mid K_{\lambda^{\prime}}^{* *}$, and therefore the residue field $K_{\lambda^{\prime}+1}^{\prime} w_{\lambda^{\prime}}=L w_{\lambda^{\prime}}$ is an algebraic extension of $K_{\lambda^{\prime}}^{* *}\left(T_{\lambda^{\prime}} \cup\left\{t_{0,0}\right\}\right) w_{\lambda^{\prime}}$. Because $\left(t_{0,0}-t_{\lambda^{\prime}+1,0}\right) w_{\lambda^{\prime}}=t_{0,0} w_{\lambda^{\prime}}$, the latter field is equal to $K_{\lambda^{\prime}}^{* *}\left(T_{\lambda^{\prime}} \cup\left\{t_{0,0}-t_{\lambda^{\prime}+1,0}\right\}\right) w_{\lambda^{\prime}}$. Again by Lemma 20, there is an algebraic extension $K_{\lambda^{\prime}}$ of $K_{\lambda^{\prime}}^{* *}\left(T_{\lambda^{\prime}} \cup\left\{t_{0,0}-t_{\lambda^{\prime}+1,0}\right\}\right)$ inside of the henselian field $\left(K_{\lambda^{\prime}+1}^{\prime}, w_{\lambda^{\prime}}\right)$ which is a field of representatives for $K_{\lambda^{\prime}+1}^{\prime} w_{\lambda^{\prime}}=L w_{\lambda^{\prime}}$. By construction, $t_{0,0}-t_{\lambda^{\prime}+1,0} \in K_{\lambda^{\prime}}$. As before, $t_{0,0} \notin K_{\lambda^{\prime}}$ as $t_{0,0}$ is transcendental over $K_{\lambda^{\prime}}^{* *}\left(T_{\lambda^{\prime}} \cup\left\{t_{0,0}-t_{\lambda^{\prime}+1,0}\right\}\right)$.

We set

$$
K:=\bigcup_{\nu<\lambda} K_{\nu}
$$

By construction, $t_{0,0} \notin K$, but $K\left(t_{0,0}\right)$ contains $t_{\nu, \mu}$ for all $\nu<\lambda$ and $\mu<\kappa_{\nu}$. Hence, $K^{\prime} \mid K\left(t_{0,0}\right)$ is algebraic and therefore, $\operatorname{trdeg} K^{\prime} \mid K=1$. With the same argument as for $K^{\prime}$, one shows that $K$ is dense in $L$. (This also follows from the fact that $t_{0,0}$ is limit of the Cauchy sequence $\left(t_{0,0}-t_{\nu+1,0}\right)_{\nu<\lambda}$ in $K$ and $K^{\prime}$ is dense in $L$.)

Now we indicate how to achieve trdeg $K^{\prime} \mid K=\lambda$. By passing to a cofinal subsequence of $\left(C_{\nu}\right)_{\nu<\lambda}$ if necessary, we can assume that every $T_{\nu}$ contains at least $|\nu|$ many elements, where $|\nu|$ denotes the cardinality of the ordinal number $\nu$. Then it is possible to re-order the elements of $T_{\nu}$ in such a way that $T_{\nu}=\left\{t_{\nu, \mu} \mid \mu<\mu_{\nu}\right\}$ where $\mu_{\nu}$ is some ordinal number $\geq \nu$. Now we modify the above construction of $K$ as follows: at every step $\nu$ where $\nu=0$ or $\nu$ is a successor ordinal, we replace $t_{\nu, \mu}$ by $t_{\nu, \mu}-t_{\nu+1, \mu}$ for all $\mu \leq \nu$. In the limit case for $\lambda^{\prime}<\lambda$, we then have that $T_{\lambda^{\prime}} \cup\left\{t_{\nu, \nu} \mid \nu<\lambda^{\prime}\right\}$ is a transcendence basis of $K_{\lambda^{\prime}}^{\prime} \mid K_{\lambda^{\prime}}^{* *}$. Here, we replace every $t_{\nu, \nu}$ for $\nu<\lambda^{\prime}$ by $t_{\nu, \nu}-t_{\lambda^{\prime}+1, \nu}$. In this way we achieve that the elements $t_{\nu, \nu}, \nu<\lambda$ will be algebraically independent over $K$, but $K$ will still be dense in $L$.

Remark 28 We can replace the field $K_{\kappa}=K\left(T \backslash T_{\kappa}\right)$ mentioned in the first paragraph of the proof by the larger field $K\left(T \backslash T_{\kappa} \backslash\{t\}\right)^{h}(t)$ where $t \in T \backslash T_{\kappa}$. By the same argument 
as given at the end of Remark 25, this field admits a truncation closed embedding into the corresponding power series field.

Propositions 23 and 27 together prove Theorem 1. Theorem 2 follows immediately from Proposition 27 since if $v L$ admits no maximal proper convex subgroup, then the cofinality of the set of convex subgroups of $v L$ is an infinite cardinal number. It remains to give the

Proof of Proposition 4: By Lemma 26 we may take a henselian valued field $(L, v)$ of residue characteristic 0 such that $v L$ admits no maximal proper convex subgroup. Using Proposition 27 we pick a non-henselian proper subfield $K$ which is dense in $L$. Lemma 16 shows that for every non-trivial coarsening $w$ of $v,(K, w)$ is dense in $(L, w)$, whence $K w=L w$. By Lemma 17, $(L w, \bar{w})$ is henselian because $(L, v)$ is henselian and $v=w \circ \bar{w}$. Hence, $(K w, \bar{w})$ is henselian, which finishes our proof.

Example 1 A more direct construction of a counterexample works as follows: Take an ascending chain of convex subgroups $C_{i}, i \in \mathbb{N}$, in some ordered abelian group. Take $k$ to be any field and set

$$
K:=\bigcup_{i \in \mathbb{N}} k\left(\left(C_{i}\right)\right) .
$$

As a union of an ascending chain of henselian valued fields, $K$ is itself a henselian valued field. But $K$ is not complete. For instance, if $0<\alpha_{i} \in C_{i} \backslash C_{i-1}$, then the element

$$
x:=\sum_{i \in \mathbb{N}} t^{\alpha_{i}} \in k\left(\left(\bigcup_{i \in \mathbb{N}} C_{i}\right)\right)
$$

lies in the completion of $K$, but not in $K$. Since every henselian field is separablealgebraically closed in its henselization (cf. [W], Theorem 32.19), $x$ is either transcendental or purely inseparable over $K$. But it cannot be purely inseparable over $K$ because if $p=$ char $K>0$, then $x^{p^{\nu}}=\sum_{i \in \mathbb{N}} t^{p^{\nu} \alpha_{i}} \notin K$ for all $\nu \geq 0$. Hence by part a) of Proposition 21, $K(x)$ (endowed with the restriction $v$ of the valuation of the completion of $K$ ) is not henselian. But for every non-trivial coarsening $w$ of $v, K(x) w=K w$ since $K$ is dense in $(K(x), v)$, and we leave it to the reader to prove that $(K w, \bar{w})$ is henselian.

\section{Small integer parts}

We will use a cardinality argument to show that there are real closed fields that are larger than the quotient fields of all its integer parts.

Lemma 29 a) Take any valued field $(L, v)$. Then all additive complements of the valuation ring of $L$, if there are any, have the same cardinality.

b) All integer parts in an ordered field, if there are any, are isomorphic as ordered sets and thus have the same cardinality. 
Proof: a): As an additive group, any additive complement of the valuation ring $\mathcal{O}$ of $L$ is isomorphic to $L / \mathcal{O}$.

b): Take two integer parts $I_{1}$ and $I_{2}$ of a given ordered field $(L,<)$. Since $I_{2}$ is an integer part, for every $a \in I_{1}$ there is a unique element $a^{\prime} \in I_{2}$ such that $a^{\prime} \leq a<a^{\prime}+1$. Hence, we have a mapping $I_{1} \ni a \mapsto a^{\prime} \in I_{2}$. Conversely, since $I_{1}$ is an integer part, there is a unique $a^{\prime \prime} \in I_{1}$ such that $a^{\prime \prime}<a^{\prime} \leq a^{\prime \prime}+1$. Consequently, $a=a^{\prime \prime}+1$ and $a$ is the only element that is sent to $a^{\prime}$, showing that the map is injective and even order preserving. On the other hand, since $a^{\prime \prime}+1$ is sent to $a^{\prime}$, the mapping is also proved to be onto.

We also need the following facts, which are well known (note that a similar statement holds for weak complements):

Lemma 30 a) If $K$ is dense in $(L, v)$, then every additive complement of the valuation ring of $(K, v)$ is also an additive complement of the valuation ring of $(L, v)$.

b) If $K$ is dense in $(L,<)$, then every integer part of $(K,<)$ is also an integer part of $(L,<)$.

Proof: We only prove a) and leave the proof of b) to the reader. Let $A$ be an additive complement of the valuation ring $\mathcal{O}_{K}$ of $(K, v)$, that is, $A \cap \mathcal{O}_{K}=\{0\}$ and $A+\mathcal{O}_{K}=K$. Denote the valuation ring of $(L, v)$ by $\mathcal{O}_{L}$. Since the valuation on $L$ is an extension of the valuation on $K$, we have that $K \cap \mathcal{O}_{L}=\mathcal{O}_{K}$ and thus, $A \cap \mathcal{O}_{L}=A \cap \mathcal{O}_{K}=\{0\}$. Now take any $a \in L$. Since $K$ is dense in $(L, v)$, there is $b \in K$ such that $v(a-b) \geq 0$, that is, $a-b \in \mathcal{O}_{L}$. Consequently, $a=b+(a-b) \in K+\mathcal{O}_{L}=A+\mathcal{O}_{K}+\mathcal{O}_{L}=A+\mathcal{O}_{L}$. This proves that $A+\mathcal{O}_{L}=L$.

We cite the following fact; for a proof, see for instance [B-K-K].

Lemma 31 If $K$ is an ordered field and $R$ is a subring which is an additive complement of the valuation ring for the natural valuation of $K$, then $R+\mathbb{Z}$ is an integer part of $K$.

For every ordered abelian group $G$, written additively, we set

$$
G^{<0}:=\{g \in G \mid g<0\} .
$$

Proposition 32 Suppose that $k$ is a countable field. Then the countable ring $k\left[\mathbb{Q}^{<0}\right]:=$ $k\left[t^{g} \mid 0>g \in \mathbb{Q}\right] \subset k((\mathbb{Q}))$ is an additive complement of the valuation ring of the uncountable henselian valued field $\operatorname{PSF}(k)$. The same remains true if $\operatorname{PSF}(k)$ is replaced by its completion.

If in addition $k$ is an ordered (respectively, real closed) field, then $k\left[\mathbb{Q}^{<0}\right]+\mathbb{Z}$ is an integer part of the ordered (respectively, real closed) field $\operatorname{PSF}(k)$, and this also remains true if $\operatorname{PSF}(k)$ is replaced by its completion. 
Proof: It is well known that every field $k((t))$ of formal Laurent series is uncountable. Hence, $\operatorname{PSF}(k)$ is uncountable. As the union of an ascending chain of fields $k\left(\left(t^{\frac{1}{n}}\right)\right)$ of formal Laurent series, which are henselian, $\operatorname{PSF}(k)$ is itself henselian. Note that the completion of a henselian field is again henselian ([W], Theorem 32.19).

Every element $a \in \operatorname{PSF}(k)$ lies in $k\left(\left(t^{\frac{1}{n}}\right)\right)$ for some $n \in \mathbb{N}$. Hence, it suffices to show that $k\left[t^{\frac{m}{n}} \mid 0>m \in \mathbb{Z}\right]$ is an additive complement of the valuation ring $k\left[\left[t^{\frac{1}{n}}\right]\right]$ in $k\left(\left(t^{\frac{1}{n}}\right)\right)$. Renaming $t^{\frac{1}{n}}$ by $t$, we thus have to show that $k\left[t^{m} \mid 0>m \in \mathbb{Z}\right]$ is an additive complement of the valuation ring $k[[t]]$ in $k((t))$. But this is clear since $k((t))$ is the set of formal Laurent series

$$
\sum_{i=N}^{\infty} c_{i} t^{i}=\sum_{i=N}^{-1} c_{i} t^{i}+\sum_{i=0}^{\infty} c_{i} t^{i}
$$

where $N \in \mathbb{Z}$ and $c_{i} \in k$. The first sum lies in $k\left[t^{m} \mid 0>m \in \mathbb{Z}\right]$ and the second sum in $k[[t]]$.

Part a) of Lemma 30 shows that $k\left[\mathbb{Q}^{<0}\right]$ is also an additive complement of the valuation ring in the completion of $\operatorname{PSF}(k)$.

The assertions about the ordered case follow from Lemma 31 together with part b) of Lemma 30.

From this proposition together with Lemma 29, we obtain the following corollary, which in turn proves Theorem 5 .

Corollary 33 Suppose that $k$ is a countable field. If $R$ is any subring which is an additive complement of the valuation ring of $\operatorname{PSF}(k)$, then Quot $R$ is countable and $\operatorname{trdeg} \operatorname{PSF}(k) \mid$ Quot $R$ is uncountable.

If in addition $k$ is an ordered field and $I$ an integer part of PSF $(k)$, then Quot $I$ is countable and $\operatorname{trdeg} \operatorname{PSF}(k) \mid$ Quot $I$ is uncountable.

The same remains true if $\mathrm{PSF}(k)$ is replaced by its completion.

Proof: The quotient field of a countable ring is again countable. So it only remains to prove the assertion about the transcendence degree. It follows from the fact that the algebraic closure of a countable field is again countable. So if $T$ would be a countable transcendence basis of PSF $(k) \mid$ Quot $R$, then $($ Quot $R)(T)$ and hence also PSF $(k)$ would be countable, which is not the case.

Denote by $k((G))=k\left(\left(t^{G}\right)\right)$ the power series field with coefficients in $k$ and exponents in $G$, and by $k(G)$ the smallest subfield of $k((G))$ which contains all monomials $c t^{g}, c \in k$, $g \in G$. Denote by $k(G)^{c}$ its completion; it can be chosen in $k((G))$. Note that the completion of $\operatorname{PSF}(k)$ is equal to $k(\mathbb{Q})^{c}$. Further, denote by $k\left[G^{<0}\right]$ the subring of $k(G)$ generated by $k$ and all monomials $c t^{g}$ where $c \in k$ and $0>g \in G$. 
Proposition 34 Suppose that $k$ is a countable field and $G$ is a countable archimedean ordered abelian group. Then the countable ring $k\left[G^{<0}\right]$ is an additive complement of the valuation ring of the uncountable henselian valued field $k(G)^{c}$.

If $R$ is any subring which is an additive complement of the valuation ring of $k(G)^{c}$, then Quot $R$ is countable and $\operatorname{trdeg} k(G)^{c} \mid$ Quot $R$ is uncountable.

If in addition $k$ is an ordered field and $I$ is an integer part of the ordered field $k(G)^{c}$, then Quot $I$ is countable and $\operatorname{trdeg} k(G)^{c} \mid$ Quot $I$ is uncountable.

Proof: By Lemma 19, $k(G)^{c}$ is henselian. (Therefore, it is real closed if and only if $k$ is real closed and $G$ is divisible.)

We show that the ring $k\left[G^{<0}\right]$ is an additive complement of the valuation ring in $k(G)$. Every element $a$ of the latter is a quotient of the form

$$
a=\frac{c_{1} t^{g_{1}}+\ldots+c_{m} t^{g_{m}}}{d_{1} t^{h_{1}}+\ldots+d_{n} t^{h_{n}}}
$$

with $c_{1}, \ldots, c_{m}, d_{1}, \ldots, d_{n} \in k$ and $g_{1}, \ldots, g_{m}, h_{1}, \ldots, h_{n} \in G$. Without loss of generality we may assume that $h_{1}$ is the unique smallest element among the $h_{1}, \ldots, h_{n}$. Then we rewrite $a$ as follows:

$$
a=\frac{\frac{c_{1}}{d_{1}} t^{g_{1}-h_{1}}+\ldots+\frac{c_{m}}{d_{1}} t^{g_{m}-h_{1}}}{1+\frac{d_{2}}{d_{1}} t^{h_{2}-h_{1}}+\ldots+\frac{d_{n}}{d_{1}} t^{h_{n}-h_{1}}} .
$$

By our assumption on $h_{1}$, all summands in the denominator except for the 1 have positive value. Hence, we can rewrite $a$ as

$$
a=\left(\frac{c_{1}}{d_{1}} t^{g_{1}-h_{1}}+\ldots+\frac{c_{m}}{d_{1}} t^{g_{m}-h_{1}}\right)\left(1+\sum_{i=1}^{\infty}(-1)^{i}\left(\frac{d_{2}}{d_{1}} t^{h_{2}-h_{1}}+\ldots+\frac{d_{n}}{d_{1}} t^{h_{n}-h_{1}}\right)^{i}\right) .
$$

In the power series determined by this geometric series, only finitely many summands will have negative value; this is true since $G$ is archimedean by hypothesis. Let $b \in k\left[G^{<0}\right]$ be the sum of these summands. Then $v(a-b) \geq 0$. This proves that $k\left[G^{<0}\right]$ is an additive complement of the valuation ring in $k(G)$. Part a) of Lemma 30 shows that $k\left[G^{<0}\right]$ is also an additive complement of the valuation ring in $k(G)^{c}$.

All other assertions are deduced like the corresponding assertions of Corollary 33.

\section{Proof of Theorem 6}

We take $k$ to be any field of characteristic 0 and

$$
L:=\bigcup_{\nu<\lambda} k\left(\left(C_{\nu}\right)\right)
$$


to be the henselian valued field constructed in Example 1. The set $\operatorname{Neg} k\left(\left(C_{\nu}\right)\right)$ of all power series in $k\left(\left(C_{\nu}\right)\right)$ with only negative exponents is a $k$-algebra which is an additive complement of the valuation ring $k\left[\left[C_{\nu}\right]\right]$ of $k\left(\left(C_{\nu}\right)\right)$. It follows that

$$
R:=\bigcup_{\nu<\lambda} \operatorname{Neg} k\left(\left(C_{\nu}\right)\right)
$$

is a $k$-algebra which is an additive complement of the valuation ring $\bigcup_{\nu<\lambda} k\left[\left[C_{\nu}\right]\right]$ of $L$. We wish to show that its quotient field is $L$. Take any element $a \in L$. Since $L$ is the union of the $k\left(\left(C_{\nu}\right)\right)$, there is some $\nu$ such that $a \in k\left(\left(C_{\nu}\right)\right)$. Pick some negative $\alpha \in C_{\nu+1} \backslash C_{\nu}$. Then $\alpha<C_{\nu}$. Denote by $t^{\alpha}$ the monic monomial of value $\alpha$ in $k\left(\left(C_{\nu+1}\right)\right)$. Then $a t^{\alpha}$ has only negative exponents, so $t^{\alpha}$ and $a t^{\alpha}$ are both elements of $\operatorname{Neg} k\left(\left(C_{\nu+1}\right)\right)$. Therefore, $a \in$ Quot Neg $\left(k\left(\left(C_{\nu+1}\right)\right) \subseteq\right.$ Quot $R$.

Now take any non-zero cardinal number $\kappa \leq \lambda$. We modify the construction in the final part of the proof of Proposition 27 in that we start with $K_{\nu}^{\prime}=k\left(\left(C_{\nu}\right)\right)$, and replace $t_{\nu, \nu}$ by $t_{\nu, \nu}-t_{\nu+1, \nu}$ (or by $t_{\nu, \nu}-t_{\lambda^{\prime}+1, \nu}$ in the limit case) only as long as $\nu \leq \kappa$. Then the elements $t_{\nu, \nu}, \nu<\kappa$, will be algebraically independent over $K=\bigcup_{\nu<\lambda} K_{\nu}$, we have $\operatorname{trdeg} L \mid K=\kappa$, and $K$ will be dense in $L$.

For every $\nu<\lambda, w_{\nu}$ induces a valuation preserving isomorphism from $\left(k\left(\left(C_{\nu}\right)\right), v\right)$ and from $\left(K_{\nu}, v\right)$ onto $\left(L w_{\nu}, \bar{w}_{\nu}\right)$. Hence, $\iota_{\nu}:=\left.\left(\left.w_{\nu}\right|_{K_{\nu}}\right)^{-1} \circ w_{\nu}\right|_{k\left(\left(C_{\nu}\right)\right)}$ is a valuation preserving isomorphism from $\left(k\left(\left(C_{\nu}\right)\right), v\right)$ onto $\left(K_{\nu}, v\right)$. For $\nu<\mu<\lambda, \iota_{\mu}$ is an extension of $\iota_{\nu}$. Hence, $\iota:=\bigcup_{\nu<\lambda} \iota_{\nu}$ is a valuation preserving isomorphism from $(L, v)$ onto $(K, v)$. The image $R_{\kappa}$ of $R$ under $\iota$ is a $k$-algebra which is an additive complement of the valuation ring of $K$ and has quotient field $K$. Consequently, $\operatorname{trdeg} L \mid$ Quot $R_{\kappa}=\kappa$. Since $K$ is dense in $L, R_{\kappa}$ is also an additive complement of the valuation ring of $L$.

If in addition $k$ is an archimedean ordered field and $<$ is any ordering on $L$ compatible with $v$, then $v$ is the natural valuation of $(L,<)$. Hence by Lemma $31, R+\mathbb{Z}$ and $R_{\kappa}+\mathbb{Z}$ are integer parts of $(L,<)$. Since Quot $(R+\mathbb{Z})=$ Quot $R$ and $\operatorname{Quot}\left(R_{\kappa}+\mathbb{Z}\right)=$ Quot $R_{\kappa}$, this completes our proof.

\section{Weak complements}

Lemma 35 Let I be an integer part of the ordered field $(K,<)$. If $v$ denotes the natural valuation of $(K,<)$, then $I$ is a weak complement in $(K, v)$.

Proof: $\quad$ Take $0<x \in K$ and assume that $v x>0$. Then for all $n \in \mathbb{N}$, also $v n x>0=v 1$ which by (1) implies that $0<n x \leq 1$. Consequently, $0<x<1$ and thus, $x \notin I$. This proves that $v r \leq 0$ for all $r \in I$.

For every $a \in K$ there is $r \in I$ such that $0 \leq a-r<1$. Again by (1), this implies that $v(a-r) \geq v 1=0$. 
In what follows, let $R$ be a weak complement in a valued field $(K, v)$. For every convex subgroup $\Gamma$ of $v K$, we define

$$
R_{\Gamma}:=\{r \in R \mid v r \in \Gamma \cup\{\infty\}\}
$$

Lemma 36 For every convex subgroup $\Gamma$ of $v K, R_{\Gamma}$ is a subring of $K$. Denote by $K_{\Gamma}$ its quotient field. Then $v K_{\Gamma}=\Gamma$.

Proof: $\quad$ Take $r, s \in R_{\Gamma}$. Then $v r, v s \in \Gamma$. Since $r-s \in R$, we have $0 \geq v(r-s) \geq$ $\min \{v r, v s\}$, showing that $v(r-s) \in \Gamma$ and thus $r-s \in R_{\Gamma}$. Further, $r s \in R$ and $v r s=v r+v s \in \Gamma$, showing that $r s \in R_{\Gamma}$. This proves that $R_{\Gamma}$ is a subring of $K$.

Since $v R_{\Gamma}:=\left\{v r \mid r \in R_{\Gamma}\right\} \subseteq \Gamma$, we know that $v K_{\Gamma} \subseteq\{\alpha-\beta \mid \alpha, \beta \in \Gamma\}=\Gamma$. On the other hand, for every $a \in K$ with $v a \in \Gamma^{<0}$ there is some $r \in R$ such that $v(a-r) \geq 0$. It follows that $v r=v a \in \Gamma$ and thus $r \in R_{\Gamma}$ and $v a=v r \in v R_{\Gamma}$. Hence, $\Gamma^{<0} \subseteq v R_{\Gamma}$, which implies that $v K_{\Gamma}=\Gamma$.

Note that $K_{\Gamma}$ is a subfield of the quotient field of $R$. Since $v K_{\Gamma}=\Gamma$, we have that $v_{\Gamma} K_{\Gamma}=\{0\}$. This means that the residue map associated with $v_{\Gamma}$ induces an isomorphism on $K_{\Gamma}$. This is in fact an isomorphism

$$
\left(K_{\Gamma}, v\right) \simeq\left(K_{\Gamma} v_{\Gamma}, \bar{v}_{\Gamma}\right)
$$

of valued fields.

Lemma 37 For every non-trivial convex subgroup $\Gamma$ of $v K$, the valued residue field $\left(K_{\Gamma} v_{\Gamma}, \bar{v}_{\Gamma}\right)$ lies dense in $\left(K v_{\Gamma}, \bar{v}_{\Gamma}\right)$.

Proof: We have to show: if $a \in K$ such that $v a \in \Gamma$, then for every positive $\gamma \in \Gamma$ such that $\gamma>v a$ there is some $b \in K_{\Gamma}$ such that $v(a-b) \geq \gamma$. Since $\Gamma^{<0} \subseteq v R_{\Gamma}$ by the foregoing lemma, we may pick some $c \in R_{\Gamma}$ such that $v c=-\gamma$. Then there is some $r \in R$ such that $v(a c-r) \geq 0$. Since $v a c=v a-\gamma \in \Gamma^{<0}$, we have $v r=v a c \in \Gamma^{<0}$ and therefore, $r \in R_{\Gamma}$. Setting $b=\frac{r}{c} \in K_{\Gamma}$, we obtain $v(a-b) \geq-v c=\gamma$.

Now we give examples for valued fields and ordered fields without weak complements or integer parts.

Basic construction: Take an arbitrary field $k$ and $t$ a transcendental element over $k$. Denote by $v_{t}$ the $t$-adic valuation on $k(t)$. Choose some countably generated separablealgebraic extension $\left(k_{1}, v_{t}\right)$ of $\left(k(t), v_{t}\right)$. Take two algebraically independent elements $x, y$ over $k(t)$. Then by Theorem 1.1 of [K1] there exists a non-trivial valuation $w$ on $K:=k(t, x, y)$ whose restriction to $k(t)$ is trivial, whose value group is $\mathbb{Z}$ and whose residue field is $k_{1}$; since $w$ is trivial on $k(t)$, we may assume that the residue map associated with 
$w$ induces the identity on $k(t)$. Now we take the valuation $v$ on the rational function field $K$ to be the composition of $w$ with $v_{t}$ :

$$
v=w \circ v_{t}
$$

Example 2 We take $k$ to be one of the prime fields $\mathbb{Q}$ or $\mathbb{F}_{p}$ for some prime $p$. We choose $k_{1}$ such that $k_{1} v_{t}=k$ and that $v_{t} k_{1} / v_{t} k(t)$ is infinite. Take $\Gamma$ to be the convex subgroup of $v K$ such that $v_{\Gamma}=w$; in fact, $\Gamma$ is the minimal convex subgroup containing $v t$.

Suppose $K$ admits a weak complement $R$. Then by Lemma 37 the isomorphic image $K_{\Gamma} w$ of the subfield $K_{\Gamma}$ of $K$ is dense in the valued residue field $\left(k_{1}, v_{t}\right)$. It follows from Lemma 12 that $v_{t}\left(K_{\Gamma} w\right)=v_{t} k_{1}$. Note that the isomorphism $K_{\Gamma} \rightarrow K_{\Gamma} w$ preserves the prime field $k$ of $K_{\Gamma}$. Since $v_{t}\left(K_{\Gamma} w\right) \neq\{0\}$, it follows from Corollary 11 that $K_{\Gamma} w$ cannot be algebraic over the trivially valued subfield $k$. Hence, $\operatorname{trdeg} K_{\Gamma} w \mid k=1$, and we take some $t^{\prime} \in K_{\Gamma}$ such that $t^{\prime} w$ is transcendental over $k$. It follows that $K_{\Gamma} \mid k\left(t^{\prime}\right)$ is algebraic. As $v_{t} k_{1} / v_{t} k(t)$ is infinite, Lemma 10 shows that $K_{\Gamma} w \mid k(t)$ and hence also $K_{\Gamma} \mid k\left(t^{\prime}\right)$ must be an infinite extension.

Since trdeg $K \mid k=3$, we have that $\operatorname{trdeg} K \mid k\left(t^{\prime}\right)=2$. Let $\left\{x^{\prime}, y^{\prime}\right\}$ be a transcendence basis for this extension. Because the algebraic extension $K_{\Gamma} \mid k\left(t^{\prime}\right)$ is linearly disjoint from the purely transcendental extension $k\left(t^{\prime}, x^{\prime}, y^{\prime}\right) \mid k\left(t^{\prime}\right)$, the extension $K_{\Gamma}\left(x^{\prime}, y^{\prime}\right) \mid k\left(t^{\prime}, x^{\prime}, y^{\prime}\right)$ is infinite. But it is contained in the finite extension $K \mid k\left(t^{\prime}, x^{\prime}, y^{\prime}\right)$. This contradiction shows that $K$ cannot admit weak complements. Note that by construction,

$$
K v=k_{1} v_{t}=k \subset K \text {. }
$$

Example 3 In the foregoing example, take $k=\mathbb{Q}$. By Lemma 8, there is an ordering $<$ on the rational function field $K=k(t, x, y)$ which is compatible with the valuation $v$. Then $(K,<)$ does not admit an integer part, because any such integer part would be a weak complement for $v$.

Example 4 In Example 2, take $k=\mathbb{Q}$. By Lemma 8 there is an ordering on $k(t)$ compatible with the $v_{t}$-adic valuation. The real closure $k(t)^{\mathrm{rc}}$ of $k(t)$ with respect to this ordering is a countably generated infinite algebraic extension of $k(t)$. So we may take $k_{1}=k(t)^{\mathrm{rc}}$. The valuation $v_{t}$ extends to a valuation of $k_{1}$ which is compatible with its ordering. Again by Lemma 8 we may choose a lifting of the ordering of $k_{1}$ to $K$ through the valuation $w$. This ordering on $K$ induces through $v=w \circ v_{t}$ the same ordering on the residue field $k$ as the ordering on $k(t)^{\text {rc }}$ induces through $v_{t}$; in particular, we find that the chosen ordering on $K$ is compatible with the valuation $v$.

Now pick any positive integer $n$ and consider the $n$-real closure $K^{\mathrm{rc}(\mathrm{n})}$ of $(K,<)$ as defined in $[\mathrm{Bg}]$. It is encluded in the real closure $K^{\mathrm{rc}}$ of $(K,<)$, so we can extend $w$ to the real closure (cf. Lemma 9) and then restrict it to $K^{\mathrm{rc}(\mathrm{n})}$; the valuation so obtained is still 
compatible with the ordering. As $K^{\mathrm{rc}} w=(K w)^{\mathrm{rc}}=k(t)^{\mathrm{rc}}=K w$ by Lemma 9, we have that $K^{\mathrm{rc}(\mathrm{n})} w=k(t)^{\mathrm{rc}}$. So $w \circ v_{t}$ is an extension of $v$ to $K^{\mathrm{rc}(\mathrm{n})}$, and we denote it again by $v$. As before, we see that it is compatible with the ordering.

Suppose $K^{\mathrm{rc}(\mathrm{n})}$ admits a weak complement $R$. We proceed as in Example 2, with $K$ replaced by $L:=K^{\mathrm{rc}(\mathrm{n})}$. As $L_{\Gamma} w$ is dense in $\left(L w, v_{t}\right)=\left(k(t)^{\mathrm{rc}}, v_{t}\right)$ we can infer from Lemma 12 that $v_{t}\left(L_{\Gamma} w\right)=v_{t} k(t)^{\mathrm{rc}}$. This in turn is the divisible hull of $v_{t} k(t)=\mathbb{Z}$ (cf. Lemma 9). Hence, $v_{t}\left(L_{\Gamma} w\right)=\mathbb{Q}$ and thus also $v L_{\Gamma}=\mathbb{Q}$. But as in Example 2 one shows that the relative algebraic closure of $k\left(t^{\prime}\right)$ in $K\left(t^{\prime}\right)$ must be a finite extension $E$ of $k\left(t^{\prime}\right)$. Now $L_{\Gamma}$ lies in the relative algebraic closure $E^{\prime}$ of $E$ in $L$, which is just the $n$-real closure of $E$. But the value group of the $n$-real closure of $E$ is the $n$-divisible hull of $v E$, which in turn is a finite extension of $v k\left(t^{\prime}\right)=\mathbb{Z}$. So the value group of $E^{\prime}$ is still isomorphic to the $n$-divisible hull of $\mathbb{Z}$. This contradicts the fact that its subfield $L_{\Gamma}$ has value group $\mathbb{Q}$. This contradiction proves that $K^{\mathrm{rc}(\mathrm{n})}$ does not admit weak complements for its compatible valuation $v$, and therefore does not admit integer parts.

In order to obtain an example where the valued field $(K, v)$ admits an embedded residue field and a cross-section, we modify Example 2 as follows.

Example 5 In our basic construction, we take $k=k_{0}(z)$ where $k_{0}$ is any prime field and $z$ is transcendental over $k_{0}$. The henselization $k_{0}(z)^{h}$ of $k_{0}(z)$ with respect to the $z$-adic valuation $v_{z}$ is a countably generated separable-algebraic extension of $k_{0}(z)$. Therefore, we may choose $k_{1}$ to be a countably generated separable-algebraic extension of $k(t)$ such that $v_{t} k_{1}=\mathbb{Z}$ and $k_{1} v_{t}=k_{0}(z)^{h}$ (cf. Theorem 2.14 of [K1]). Then we take

$$
v^{\prime}=v \circ v_{z}=w \circ v_{t} \circ v_{z} .
$$

Let $\Gamma$ be the convex subgroup of $v^{\prime} K$ such that $v_{\Gamma}^{\prime}=w$; now $\Gamma$ is the minimal convex subgroup containing $v^{\prime} t$. Suppose that $\left(K, v^{\prime}\right)$ admits a weak complement $R$. Then by Lemma 37 the isomorphic image $K_{\Gamma} w$ of the subfield $K_{\Gamma}$ of $K$ is dense in the valued residue field $\left(k_{1}, v_{t} \circ v_{z}\right)$. The isomorphism $K_{\Gamma} \rightarrow K_{\Gamma} w$ preserves the prime field $k_{0}$ of $K_{\Gamma}$. From Lemma 12 we infer that $v_{t} \circ v_{z}\left(K_{\Gamma} w\right)=v_{t} \circ v_{z}\left(k_{1}\right)$. This value group has two non-trivial convex subgroups, namely, itself and the smallest convex subgroup which contains $v_{t} \circ v_{z}(z)$. We choose elements $t^{\prime}, z^{\prime} \in K_{\Gamma}$ such that $v_{t} \circ v_{z}\left(t^{\prime} w\right)>0$ lies in the former, but not in the latter, and $v_{t} \circ v_{z}\left(z^{\prime} w\right)>0$ lies in the latter. Then these two values are rationally independent. Thus by Theorem 1 of $[\mathrm{Br}]$, Chapter VI, $\S 10.3, t^{\prime} w, z^{\prime} w$ are algebraically independent over the trivially valued field $k_{0}$. But as $K_{\Gamma} w \subseteq k_{1}$, we must have trdeg $K_{\Gamma} w \mid k_{0}=2$. Hence $K_{\Gamma} w \mid k_{0}\left(t^{\prime} w, z^{\prime} w\right)$ is algebraic, and so is $K_{\Gamma} \mid k_{0}\left(t^{\prime}, z^{\prime}\right)$.

By Lemma $16, K_{\Gamma} w$ is also dense in $\left(k_{1}, v_{t}\right)$. Hence $\left(K_{\Gamma} w\right) v_{t}=k_{1} v_{t}=k_{0}(z)^{h}$ by Lemma 12. Hence, $z \in\left(K_{\Gamma} w\right) v_{t}$ and we can in fact choose $z^{\prime}$ such that $\left(z^{\prime} w\right) v_{t}=z$. Consequently, $k_{0}\left(t^{\prime} w, z^{\prime} w\right) v_{t}=k_{0}(z)$ (cf. the already cited Theorem 1 of [Br]). Since $\left(K_{\Gamma} w\right) v_{t}=k_{0}(z)^{h}$ is an infinite extension of $k_{0}(z)$ by part a) of Proposition 21, it follows from Lemma 10 that $K_{\Gamma} w$ is an infinite extension of $k_{0}\left(t^{\prime} w, z^{\prime} w\right)$. Thus, $K_{\Gamma}$ is an infinite extension of $k_{0}\left(z^{\prime}, t^{\prime}\right)$. 
Since trdeg $K \mid k_{0}=4$ and $\operatorname{trdeg} k_{0}\left(z^{\prime}, t^{\prime}\right)\left|k_{0}=\operatorname{trdeg} k_{0}\left(z^{\prime} w, t^{\prime} w\right)\right| k_{0}=2$, we have that $\operatorname{trdeg} K \mid k_{0}\left(z^{\prime}, t^{\prime}\right)=2$. Let $\left\{x^{\prime}, y^{\prime}\right\}$ be a transcendence basis for this extension. Because the algebraic extension $K_{\Gamma} \mid k_{0}\left(z^{\prime}, t^{\prime}\right)$ is linearly disjoint from the purely transcendental extension $k_{0}\left(z^{\prime}, t^{\prime}, x^{\prime}, y^{\prime}\right) \mid k_{0}\left(z^{\prime}, t^{\prime}\right)$, the extension $K_{\Gamma}\left(x^{\prime}, y^{\prime}\right) \mid k_{0}\left(z^{\prime}, t^{\prime}, x^{\prime}, y^{\prime}\right)$ is infinite. But it is contained in the finite extension $K \mid k_{0}\left(z^{\prime}, t^{\prime}, x^{\prime}, y^{\prime}\right)$. This contradiction shows that $\left(K, v^{\prime}\right)$ cannot admit weak complements.

The value group $v^{\prime} K$ is the lexicographic product $w K \times v_{t} k_{1} \times v_{z} k_{0}(z) \simeq \mathbb{Z} \times \mathbb{Z} \times \mathbb{Z}$ since $v_{z} k_{0}(z)=v_{z} k_{0}(z)^{h}=\mathbb{Z}$. This shows that $\left(K, v^{\prime}\right)$ admits a cross-section. The residue field $K v^{\prime}=k_{0}$ is embedded in $K$. Note that $K$ is a rational function field of transcendence degree 4 over its residue field.

Example 6 In the foregoing example, take $k_{0}=\mathbb{Q}$. By Lemma 8, there is an ordering $<$ on the rational function field $K=k(t, x, y, z)$ which is compatible with the valuation $v^{\prime}$. Then $(K,<)$ does not admit an integer part. Nevertheless, the valuation $v^{\prime}$, which is the natural valuation of the ordering $<$ since $K v^{\prime}=\mathbb{Q}$ is archimedean ordered, admits an embedding of its residue field and a cross-section.

Finally, let us note that Proposition 21 shows:

Proposition 38 None of the valued fields in the above examples are henselian. Also, the natural valuation of the example constructed by Boughattas in [Bg] is not henselian.

Proof: It follows from part a) of Proposition 21 that the rational function fields of Examples 2 and 3 are not henselian. The fields of Example 4 and Boughattas' example are $n$-real closures of algebraic function fields. The " $n$-algebraic closures" of part b) of Proposition 21 are algebraic extensions of the $n$-real closures. Since they are not henselian, Lemma 15 shows that the same holds for the $n$-real closures.

\section{References}

[B-K-K] Biljakovic, D. - Kotchetov, M. - Kuhlmann, S.: Primes and irreducibles in truncation integer parts of real closed fields, this volume

[Bg] Boughattas, S.: Résultats optimaux sur l'existence d'une partie entière dans les corps ordonnés, J. Symb. Logic 58 (1993), 326-333

[Br] Bourbaki, N.: Commutative algebra, Paris (1972)

[E] Endler, O.: Valuation theory, Berlin (1972)

[Fo] Fornasiero, A. : Embedding Henselian fields in power series, preprint

[Fu] Fuchs, L. : Partially ordered algebraic systems, Pergamon Press, Oxford (1963)

[K1] Kuhlmann, F.-V.: Value groups, residue fields and bad places of rational function fields, Trans. Amer. Math. Soc. 356 (2004), 4559-4600 
[K2] Kuhlmann, F.-V.: Book in preparation. Preliminary versions of several chapters available at: http://math.usask.ca/ fvk/Fvkbook.htm

[KS] Kuhlmann, S.: Ordered Exponential Fields, The Fields Institute Monograph Series, vol. 12, AMS Publications (2000)

[L] Lang, S.: The theory of real places, Ann. of Math. 57 (1953), 378-391

[M-R] Mourgues, M. - H. - Ressayre, J.- P.: Integer parts Every real closed field has an Integer Part, Journal of Symbolic Logic, 58 (1993), 641-647

[M-S] MacLane, S. - Schilling, O.F.G.: Zero-dimensional branches of rank 1 on algebraic varieties, Annals of Math. 40 (1939), 507-520

[N] Neukirch, J.: Algebraic number theory, Springer, Berlin (1999)

[P] Prestel, A.: Lectures on Formally Real Fields, Springer Springer Lecture Notes in Math. 1093, Berlin-Heidelberg-New York-Tokyo (1984)

[R] Ribenboim, P.: Théorie des valuations, Les Presses de l'Université de Montréal (1964)

[W] Warner, S. : Topological fields, Mathematics studies 157, North Holland, Amsterdam (1989)

[Z-S] Zariski, O. - Samuel, P.: Commutative Algebra, Vol. II, New York-Heidelberg-Berlin (1960)

Mathematical Sciences Group, University of Saskatchewan,

106 Wiggins Road, Saskatoon, Saskatchewan, Canada S7N 5E6

email: fvk@math.usask.ca 